\title{
Fremd im Eigenen - Hemmungen in der Arbeit mit Gewalterfahrenen ${ }^{1}$ \\ Elizabeth Högger Klaus (Zürich), Sophie Schneider (Zürich), Michael Niebler (Zürich)
}

Zusammenfassung: Gewalt ist allgegenwärtig. Nicht nur die Nachrichten berichten täglich davon, vielmehr leben Zeugen von Gewalt in Form von Ausgrenzung, Folter, Rassismus, behördlicher Willkür, Massenmorden usw. unter uns. Dennoch gibt es eine grosse Hemmung, sich mit den Menschen, die Gewalt erfahren haben, auseinanderzusetzen. Ein Grund dafür sind schwer erträgliche Gegenübertragungen als Reaktion auf chaotische Phänomene, da die innerpsychische Ordnung auf vielfältige Weise zerstört ist. Geordnetes Zeiterleben geht in Teilen verloren und das Grundvertrauen in sich und den Anderen wird angegriffen. Der immer auch in der psychoanalytischen Situation enthaltene Bruch, das Gegenüber als stets fremd erleben zu müssen, verweist uns auch auf das Fremde in uns. Die Gewalt radikalisiert dieses Erleben, lässt unmöglich erscheinen, den Anderen zu verstehen. Stammt das Gegenüber auch noch aus einem anderen Kulturkreis, ist er uns buchstäblich fremd. Es muss die Spannung zwischen widersprüchlichen Weltanschauungen ausgehalten werden, ohne einem Ordnungssystem den Vorzug zu geben. Für die therapeutische Arbeit ist es wesentlich, diese neutrale Position einzuhalten, um als Zeuge für das Erleben des Patienten zurVerfügung zu stehen. Wir illustrieren dies unter Einbezug der interkulturellen Perspektive durch die Beschäftigung mit dem Roman «Rückkehr nach Haifa» von Ghassan Kanafãni, der Untersuchung eines fremden Krankheitsverständnisses (am Beispiel Kambodscha) und einem Fallbeispiel eines südafrikanischen Mannes.

Schlüsselworte: Gewalterfahrung, Zeiterleben, Fremdheit, Zeugenschaft

... [D]er Verstehensprozess [gleicht] nicht zwei hell erleuchteten Zügen, die nebeneinander herfahren, sondern eher zwei einander begegnenden Fahrzeugen, die für einen Moment optisch verkoppelt sind, um dann wieder in entgegengesetzte Richtungen zu rasen. (Hahn, 1997, S. 136) 
Man mag sich entsprechend fragen, ob ein gegenseitiges Verstehen überhaupt möglich ist. Im Alltag wie auch in der psychoanalytischen Gesprächssituation meinen wir einander sehr wohl zu verstehen, als gingen wir alle immer vom gleichen aus. Dieses implizite Weltwissen, auf denen unsere je eigene Weltsicht beruht und «von dem wir im Alltag erst einmal ausgehen und von dem wir handelnd unterstellen, dass auch die anderen es tun» (Hahn, 1997, S. 146), ist nicht voll begründbar und zugleich nur schwer anfechtbar. Auf die Probe gestellt werden unsere Grundannahmen, wenn wir mit Menschen aus verschiedenen Kulturkreisen arbeiten: Man wird gezwungen, mit etwas Fremdem in Kontakt zu treten, das auf das Fremde in einem selbst verweist (vgl. Kristeva, 1990). Dass wir als PsychoanalytikerInnen diese Kränkung der eigenen Weltsicht geradezu wünschen, ist nicht der einzige Widerspruch. «Psychoanalyse sucht mit Sprachmitteln das Nichtsprachliche zu erkunden. Oder, anders ausgedrückt: Sie sucht das Nichtverständliche zu verstehen» (Lorenzer, 1983, S. 97). Wie können wir das Fremdseelische psychoanalytisch überhaupt verstehen?

... das Verstehen des Fremden tastet sich an dem entlang, was ich von mir selber kenne... Ich gehe mit meiner Eigenperspektive, meinem Vor-Urteil [Gadamer (2010, S. 275); Anm. d. Verf.] ... an den Text heran und muss regelmässig erleben, dass das Vorurteil scheitert, der Text anderes meint, als ich dachte ... bis ich an den Punkt komme, wo das Vorurteil scheitert, wo ich auf das Fremde, von meinem Vorverständnis her nicht mehr Erklärbare stosse, wo das Fremde sein Recht verlangt und ich vom Patienten lerne. (Bittner, 1993, S. 204-205)

Waldenfels zufolge (1999, S. 74) gehört zur Differenz von Eigenem und Fremden eine unaufhebbare Präferenz des Eigenen «im Sinne eines Sichunterscheidens, eines Selbstbezugs in der Beziehung, der dem Verhältnis zwischen Ich und dem Anderen, zwischen Eigen- und Fremdkultur eine unaufhebbare Asymmetrie [Hervorh. im Original] verleiht.» Letztlich können wir das Fremde in seiner Andersheit nicht aus Eigenem erraten. Wir sind vielmehr auf die Hilfe des Fremden angewiesen. Denn wie das Rumpelstilzchen heisst, kann es nur selbst sagen. Küchenhoff betont ebenfalls den Aspekt der Intersubjektivität, wenn er meint: „Es gibt nicht den Text, den nur einer produziert - und der andere hat nur zu deuten. Vielmehr stricken beide am Text, den sie dann miteinander zu verstehen haben“ (2015, S. 30). Erschwert bleibt ein Verstehen insbesondere bei Erlebnissen 
seitens Patienten, «die so verstörend sind, dass ein Sprechen darüber kaum gelingen will» (Kraus, 2014, S. 109). So drücken sich unverarbeitete Gewalterfahrungen vielmehr durch ein Fehlen, eine Leerstelle im Sprechen aus. Henningsen spricht von einem Zustand der Entleerung als einer Erfahrung seelischer Nichtexistenz. «Es handelt sich um existentielle Grenzerfahrungen, für die es keine Worte gibt» (2012, S. 224). Entsprechend gross sind die Hemmungen, sich auf einen Prozess mit einem Menschen einzulassen, der Gewalt erfuhr und nicht integrieren konnte.

Stammt das Gegenüber noch dazu aus einem uns fremden Kulturkreis, verstärkt sich die Hemmung vor dem Fremden - nicht zuletzt deshalb, weil unsere kulturell konstruierten Weltanschauungen in Frage gestellt werden. Ottomeyer \& Renner (2008, S. 165)sprechen von einer «doppelt befremdlichen Begegnung» bei der Arbeit mit Menschen, welchen nebst der Gewalt via Trauma zusätzlich kulturelle Umbrüche zu schaffen machen. Wie wir exemplarisch zu erfassen versuchen, sind darüber hinaus Grundkonzepte wie Scham, Schuld, Verantwortung, Freiheit usw. je nach kulturellem Kontext anders aufzufassen, da unterschiedlich konstruiert (vgl. Kimura, 1995). Dies sind allesamt Faktoren, welche zur Verunsicherung der AnalytikerIn beitragen und ein zusätzliches Hemmnis bedeuten können in der Arbeit mit Menschen unterschiedlicher Ethnien.

Halten wir fest, dass sowohl Gewalterfahrungen als auch ein anderer kultureller Hintergrund erheblichen Einfluss auf unseren Umgang, wie wir dem Fremden begegnen, haben. Wir meinen, dass sich genannte Effekte sogar multiplizieren und die Hemmung erklären, welche wir spüren, wenn wir mit gewalterfahrenen Menschen, die aus einem anderen Kulturkreis stammen, in Kontakt treten. Im Folgenden wollen wir unsere Thesen aus verschiedenen Blickwinkeln zu erkunden versuchen. Dabei basiert dieser Artikel auf einem Seminar des PSZ in Zusammenarbeit mit Entresol (Netzwerk für Wissenschaften der Psyche), das die beiden Autorinnen zusammen mit Daniel Strassberg im Sommersemester 2018 abhielten. Das behandelte Material stellt eine Auswahl und behandelt das vielschichtige Thema nicht umfassend.

\section{Störungen des Zeiterlebens}

Als Alternative zu Fallgeschichten bieten literarischeWerke die Möglichkeit, sich einem Phänomen zu nähern. Wir meinen auf ein Werk zurückgreifen zu können, das durch seine Qualität einen guten Zugang zur Thematik eröffnet, insbesondere um den Teilaspekt der Störung des Zeiterlebens demonstrieren zu können.

Ghassan Kanafãni widmet sich in seinem Roman «Rückkehr nach Haifa» dem schwierigen Verhältnis von Arabern und Juden im neu gegründeten 
Israel. Den historischen Hintergrund des Romans bildet der Rückzug der britischen Mandatsmacht aus Palästina 1948 (für das Folgende: Beil, 2013). Dieses Machtvakuum versuchten die v. a. aus Europa zugewanderten Juden wie arabische Palästinenser gleichermassen zu nutzen. Noch vor der offiziellen Gründung von Israel am 14. Mai 1948 und dem parallelen Rückzug der britischen Truppen, begann eine Offensive durch die «Haganah» (hebräisch für «Die Verteidigung», eine paramilitärische zionistische Untergrundarmee, die nach der Staatsgründung in die israelische Verteidigungsstreitkräfte überführt wurde). Diese hatte das Ziel, die dem neu zu gründenden Staat Israel durch die UNO-Vollversammlung zugesprochenen Gebiete zu sichern. Dabei wurden allein aus Haifa 60000 Palästinenser vertrieben.

Die Protagonisten des Romans sind Said und dessen Ehefrau Safija. Beide werden am 21. April 1948 aus Haifa vertrieben. Während Said vergeblich versucht zu seiner Frau zu gelangen, geht Safija ohne ihren fünfmonatigen Sohn Chaldun aus dem Haus und kann wegen der vorrückenden Haganah-Truppen nicht mehr zurückkehren. Das Paar muss Haifa zu zweit verlassen und lebt fortan in Ramallah. Der Roman setzt ein, als sich beide am 30. Juni 1967 erstmals nach fast 20 Jahren auf den Weg nach Haifa machen. Wenngleich die Möglichkeit zur Rückkehr schon lange bestand, kommt der Entschluss zu dieser Reise am Abend bevor sie aufbrechen, angeregt durch Safija. Said reagiert zunächst abwehrend, bis er es sich nach einer langen Nacht anders überlegt.

Als er ins Bett ging, war ihm - tief im Innersten - klar, dass es kein Entrinnen gab, dass der schon zwanzig Jahre lang vorhandene Gedanke geboren war und sich auf keine Weise wieder begraben liess. Doch obwohl er wusste, dass seine Frau nicht schlief, sondern die ganze Nacht an dasselbe dachte wie er, sprach er kein Wort mit ihr. (Kanafãni, 1992, S. 32)

Hier zeigt sich die Unfähigkeit der Protagonisten, über die unglaublichen Geschehnisse zu sprechen, diese tauchen nur langsam bruchstückhaft, in Rückblenden auf, dann aber mit voller Wucht: «Plötzlich kam die Vergangenheit scharf wie ein Messer» (ebd. S. 15). Die Autoreise an die altbekannten Orte geht weiter und immer wieder bricht «... die Vergangenheit hervor wie ein Vulkan ...» (S. 13) und Said kann nicht mehr zwischen damals und heute unterscheiden «... als wäre er nicht diese zwanzig bitteren Jahre weg gewesen!» (S. 14) Dieses Verschmelzen von damals und heute wird in eindrücklichen Bildern beschrieben, als sich das Ehepaar langsam und wie unwillkürlich ihrem alten Haus nähert. Immer ist Said 
dem Erleben hilflos ausgeliefert und muss passiv erleiden, wenn «... lärmend die Vergangenheit mit all ihren Schrecken auf[taucht]» (S. 15). Ausgelöst durch ihm von früher bekannte Orte und Namen verliert Said dann die Orientierung:

Vergangenheit und Gegenwart überlagerten sich, und beide griffen ineinander mit Gedanken und Vorstellungen, Phantasien und Gefühlen, die ihm seit zwanzig Jahren folgten ... Manchmal sagte er sich:Ja, ich habe es gewusst, noch bevor es geschah. Und manchmal sagte er sich: Nein, ich bilde es mir erst ein, seit es geschehen ist. Ausgeschlossen, dass ich etwas so Schreckliches erwartet hätte. (S. 19)

Mit dem Schrecklichen ist Saids Ringen gemeint, nach Hause zu seiner Frau und dem neugeborenen Sohn zu gelangen, jedoch vor den vorrückenden Truppen und Flüchtlingsströmen zurückweichen zu müssen. Eindrücklich erscheint das Verwischen von Zukunft und Vergangenheit bei der psychischen Verarbeitung einer nicht in Worte zu fassenden Erfahrung von Gewalt und einem dadurch erlittenen Verlust. Winnicott spricht in seiner Untersuchung der «Angst vor dem Zusammenbruch» (1991) davon, dass seine Patienten mit dieser existentiellen Furcht vor etwas Kommendem, eigentlich einen bereits erlittenen Zusammenbruch, der noch vor der expliziten Erinnerungsfähigkeit liegt, meinen. Entsprechend könnte man sagen, dass das Unaussprechliche von derVergangenheit in die Zukunft verlagert wurde. Parallel zu Said erlebt auch Safija das Herumirren zwischen Soldaten und Beschuss wieder. Es scheinen «Generationen und Zeitalter» (S. 25) zu vergehen, bis sich das Ehepaar doch findet und beide schliesslich «zu keinem Gefühl fähig»(S. 26) die Stadt ohne ihr Kind verlassen müssen.

Als LeserIn erlebt man unwillkürlich das Verschwimmen der Erzählgegenwart mit den in Rückblenden erzählten Erinnerungen mit «... als seien die vergangenen zwanzig Jahre in eine gigantische Presse gelegt und zu einem kaum mehr sichtbaren hauchdünnen Blatt zusammengepresst ...» (S. 36) und gleichsam auf die Buchseite gedruckt worden. Als gleichzeitig irritierende und haltgebende Erzählelemente tauchen immer wieder nur scheinbar nebensächliche Fakten auf. So erfährt man, welche Automarken Said damals (Ford Baujahr 1946) und heute (Fiat) fährt. Während der Autofahrt durch das Haifa, in das sie zurückkehren, werden die Veränderungen aufmerksam beobachtet und gleichzeitig zu verleugnen versucht. Als sie vor ihrem ehemaligen Haus ankommen, stellt Said fest: «Sie haben die Klingel geändert.» (S. 37), erst danach erscheint das eigentlich Wesentliche, wenn er fortfährt: «Den 
Namen natürlich auch.» Erzähler wie Protagonisten müssen sich an konkretistisch anmutenden Fakten festhalten, sonst würde sie die oben genannte Wucht der Vergangenheit fortreissen und einer stringenten Logik berauben. Wie Kraus ausführt, stellen langandauernde Erlebnisperioden, die in Verbindung mit Traumata stehen eine grosse Schwierigkeit für die Erzählbarkeit dar und müssen daher in Einzelepisoden erzählt werden (2014, S. 238).

Gutwinski-Jeggle beschäftigt sich damit, wie Traumata auf das Zeiterleben wirken. Ausgangspunkt ist Freuds Feststellung: «Wir haben erfahren, dass die unbewussten Seelenvorgänge an sich zeitlos sind» (Freud, 2000, S. 238).

\begin{abstract}
Dadurch sind unbewusste Ereignisse unbeeinflussbar von Zeit. Dies ist vor allem für solche Entwicklungsgeschichten relevant, die durch Traumata pathologisch veränderte Verläufe genommen haben. Durch Verdrängung oder Abspaltung werden unerträgliche Erlebnisse vom Bewusstsein ferngehalten und ins Unbewusste verbannt. Dort führen sie aber dennoch ein wirksames Eigenleben. Nicht verankert in der fortschreitenden Zeit, bäumen sie sich, stehenden Wellen vergleichbar, im Rhythmus des Wiederholungszwanges auf... Unbewusste Probleme können nicht altern und nicht vergehen. (Gutwinski-Jeggle, 2017, S. 158)
\end{abstract}

Zeiterleben ist keine von Anfang gegebene Wahrnehmungsdimension, sondern muss sich erst im Laufe der Kindheit entwickeln und betrifft vor allem Bewusstes wie Vorbewusstes. Mit Bion und Freud geht Gutwinski-Jeggle davon aus, dass sich Denken, also ein Umgang mit Abstraktem, sozusagen als Notlösung aus der Abwesenheit des Objekts ergibt (vgl. ebd. S. 168 ff.). Ist die Spannung durch die Abwesenheit zu gross, kann kein Denken entstehen und es kann keine Abstraktion erreicht werden. Das Erleben bleibt an den konkreten Dingen haften, ein zweiter Raum für Symbolisches, der über das unmittelbar Sichtbare hinausverweisen würde, kommt nicht zustande.

An dieser Stelle schliessen wir die Romanbetrachtung ab, um ein Zwischenfazit zu ziehen. Gewalterfahrungen führen zu einer Störung der innerpsychischen Zeitordnung. Vergangenheit, Gegenwart und Zukunft geraten durcheinander. Die Betroffenen suchen deswegen Halt in einem Haften an konkreten Dingen. Für die analytische Situation bedeutet dies eine erhebliche Irritation. Um dennoch zu einem Verstehen zu gelangen, bedarf es einer hohen Toleranz für das unverständliche konkretistische Sprechen der PatientIn. 
Eine weitere Herausforderung ist das Aushalten des Fremden und die Notwendigkeit kulturspezifischer Kenntnisse, was anhand der Fallvignette im nächsten Abschnitt gezeigt werden soll.

\section{Begegnungen mit dem Fremden}

Der im Folgenden geschilderte Fall stammt aus der Zeit, in der Elizabeth Högger Klaus in Kambodscha gelebt und an der Universität Phnom Penh gearbeitet hat.

M. sucht mich auf Anraten ihrer Freundin zu einem Therapiegespräch auf. Sie ist eine 30-jährige Frau mit ebenmässigen Gesichtszügen, langen schwarzen Haaren und hellbrauner Haut. Ihr Blick ist offen, wach und erwartungsvoll. Wir sprechen miteinander auf Englisch, einer Fremdsprache für Patientin und Therapeutin. Im Zeitraum von anderthalb Jahren werden es sieben Gespräche sein. Dies scheint aus «westlicher» Sicht eine sehr niedrige Frequenz, die mit einem «Widerstand» erklärt werden könnte. Demgegenüber steht jedoch ein anderer Umgang mit Zeit. Kambodscha ist ein Land mit einer sehr hohen Zahl an Feiertagen (insgesamt 27, die vorher und nachher jeweils um Tage verlängert werden), weshalb eine Regelmässigkeit und ein Wochenrhythmus auch in anderen Kontexten als dem therapeutischen nur sehr schwer herzustellen ist.

M. erzählt mir, dass sie in den Vorlesungen immer wieder einschlafe. Sie könne nachts nicht durchschlafen, habe Albträume und sei tagsüber oft müde. Sie fühle sich unwohl und ihr sei oft schwindelig. Sie habe zunächst ein naturwissenschaftliches Fach studiert, dann aber zu einem sozialwissenschaftlichen Studiengang gewechselt. Ihre Grossmutter väterlicherseits sei gänzlich dagegen, dass sie studiere. Ihr Vater äussere sich dazu sehr ambivalent. Auch er habe deswegen Albträume.

Zunächst sprechen wir über die konkreten Schlafverhältnisse. M. erzählt mir, dass sie mit allen Familienmitgliedern in einem Raum schlafen würde. Das sind Vater, Mutter, zwei Brüder und vier Schwestern. Sie sei die Älteste. Die anderen Familienmitglieder würden oft noch fernschauen, wenn sie schon schlafen gehen wolle.

Des Weiteren trage sie die Verantwortung sowohl für ihre Geschwister als auch für ihre Cousins. Der Vater übergebe ihr die Rolle des Familienoberhaupts, wenn er abwesend sei und auf dem Feld arbeite: Sie sei dann «wie die Königin über die ganze Familie, mit der Verantwortung für alle». Das sei sehr belastend für sie. Zusätzlich würden auch die Verwandten, wenn sie vom Land in die Stadt kämen um bspw. zum Arzt zu gehen, selbstverständlich bei ihnen wohnen und essen. So 
habe sie oft zusätzliche Aufgaben. Da soziale Strukturen fast ausschliesslich über die Familie definiert sind, wird innerhalb der Familie erwartet, dass finanzielle Unterstützung gewährleistet wird (vgl. Heuveline, 2017). Die Mutter beklage diese Umstände; der Vater dulde sie und heisse die Mutter «ruhig zu bleiben».

Was M. am meisten belastet, sei aber, dass sie seit ihrer Kindheit von ihrer Familie für den Tod einer Tante verantwortlich gemacht werde. Sie habe als Kind gehört, wie ihre Tante ihren Eltern und der Grossmutter erzählt habe, dass sie vom Ehemann vergewaltigt worden sei, und davon schwanger geworden sei. M. sei dann zu diesem Onkel gelaufen und habe ihm erzählt, was sie gehört habe. Dieser habe daraufhin seine Frau geschlagen. Als die Tante das Kind dann zur Welt gebracht habe, sei sie verblutet. Die Grossmutter habe gesagt, das liege daran, dass M. ein Geheimnis weitererzählt habe, was sie nicht hätte machen dürfen. Für Mädchen gibt es in Kambodscha einen seit langem tradierten Verhaltenskodex "Chbab Srey», der immer noch Gültigkeit hat (Nakagawa, 2006). Lange habe sie daran geglaubt und habe schwer daran getragen. Später habe ihre Mutter ihr erzählt, dass die Hebamme bei der Entbindung notwendige Handlungen unterlassen habe, weil die Tante bei der Geburt ihres ersten Kindes nicht genügend Opfergaben gegeben habe. Wie mir die Patientin mitteilte, wären die Opfergaben für die Götter dann als Lohn an die Hebamme gegangen.

M. habe seit ihrer Kindheit grosse Mühe, über persönliche Probleme zu reden. Sie habe stets grosse Angst, etwas falsch zu machen. Sie sei wütend auf ihren Vater, dass er nicht auf ihrer Seite stehe, sondern der «anderen Familienseite» glaube, die ihr diese Schuld anlasten würde.

Über das Schicksal der Familie in der von Gewalt geprägten Vergangenheit des Landes erzählt die Patientin nichts. M. sagt auf Nachfrage, sie wisse nicht mehr darüber und habe mit den Eltern kaum je darüber gesprochen. Mit grosser Wahrscheinlichkeit ist jedoch davon auszugehen, dass die Eltern mit Gewalt als Täter und/oder Opfer konfrontiert worden sind, da die gesamte Bevölkerung, die nicht ins Ausland geflohen war, von den Khmer Rouge in Arbeitslagern gefangen gehalten worden war (Chandler, 1991).

Ich bin zunächst sehr verwirrt, als ich M. zuhöre. Es entsteht ein Gefühl von Unsicherheit, ob ich die Geschichte, die sie von ihrer Tante erzählt, glauben kann. Es macht mir Angst, dass einer Frau angeblich die Hilfe verweigert worden war, weil sie «den Ahnen nicht genügend Respekt gezeigt hatte» bzw. mit dem Leben zahlen musste, weil sie eine frühere Behandlung nicht ausreichend bezahlt hatte. Ich suche nach dem Vertrauten in der Begegnung und merke, dass ich über die Frage der «Schuld» nachzudenken beginne. 
Ungeachtet dessen biete ich ihr etwas anderes an. Ich überlege mit ihr, wie sie ihre Schlafbedingungen verbessern könnte. Sie nimmt sich vor, mit den Familienmitgliedern bezüglich des abendlichen Fernsehens zu reden. Insbesondere zeige ich ihr aber Entspannungsübungen (Atem- und Achtsamkeitsübungen) in der Hoffnung, dies helfe bezüglich des Einschlafens. M. ist sofort begeistert und erzählt mir bei der nächsten Sitzung, dass dies sehr gut funktioniert habe. Auch habe sie mit ihrer Familie geredet und es sei abends nun ruhiger. Sie könne besser einschlafen.

Die Entspannungsübungen hatte ich spontan gewählt, um ihr etwas anbieten zu können. Da Beziehungen durch Transaktionen bestimmt werden, wusste ich, dass es wichtig ist, etwas Konkretes aus einer Sitzung «mitzugeben», ähnlich wie beim Aufsuchen eines buddhistischen Mönches z. B. ein Armband, beim Kru Khmer ein Amulett und beim Arzt ein Medikament mitgegeben wird (Ovesen \& Trankell, 2010).

In den weiteren Gesprächen geht es vor allem um die enorme Verantwortungsübernahme und die dadurch entstehende Belastung von M. Sie beschäftigt sich in der Therapie mit den Schwierigkeiten, sich unter dem grossen Druck der Erwartungen einer bestimmten Rollenübernahme als Familienoberhaupt und Stellvertreterin des Vaters ihren persönlichen Raum nehmen zu können und formuliert für sich, dass sie gern mehr Zeit für sich hätte. So steht auch die Entscheidung, zu studieren im Spannungsfeld, das zwischen den Erwartungen der Familie, insbesondere der Grossmutter, und ihren eigenen Interessen aufgespannt wird. Sie nimmt sich vor, mit den Eltern darüber zu sprechen. Wie sie in der darauffolgenden Sitzung berichtet, ist sie hier ebenfalls auf Verständnis gestossen. Sie könne sich erlauben mehr Zeit für sich zu nehmen, um sich zwischendurch auch erholen zu können.

Ich staune über die raschen Erfolge, bin aber auch unsicher, ob sie nicht nur berichtet werden, um mich zufrieden zu stellen. Möglicherweise spielt die hierarchische Beziehung hier eine Rolle: Vielleicht gelingt es M. besser, ihre Wünsche in der Familie zu kommunizieren, wenn sie weiss, dass sie von einer angesehenen Person (Zuschreibungen aufgrund meiner Rolle an der Universität) unterstützt werden?

Meine therapeutische Hypothese ist zunächst, dass die Schwierigkeiten, den eigenen Raum zu finden, durch die Schuldzuweisungen durch die Familie am Tod ihrer Tante, weil sie über etwas gesprochen hatte, verstärkt werden. M. äussert in der Therapie zwar die Überzeugung, dass die Tante durch die unterlassenen Handlungen der Hebamme gestorben war. Dies könnte als naturwissenschaft- 
lich-medizinische Sichtweise gedeutet werden, die der traditionellen Sichtweise, in der Geister, rituelle Handlungen sowie traditionelle Verhaltenscodices und Karma eine Rolle spielen, entgegensteht. Sie könnten aber auch auf eine kambodschanische Vorstellung davon, wie M.'s körperliche Symptome wie Albträume, Schwindel und Einschlafen am Tage entstehen, hinweisen. M. spricht nicht über diese Vorstellungen, vermutlich, weil sie kulturimmanent sind. Dazu ist ein Hinweis auf das Verständnis der Selbst-Konzeption und die kambodschanische Psychophysiologie hilfreich.

In der kambodschanischen Vorstellung von der Entstehung von Krankheiten spielen - wie in vielen asiatischen Kulturen - Winde eine grosse Rolle, die, wenn sie blockiert werden, verschiedene Symptome verursachen können. So leiden KambodschanerInnen oft an Schmerzen in den Gelenken, da die Vorstellung besteht, die Winde seien dort blockiert. Kniee oder Ellbogen werden dann mit Ölen massiert, oder es wird mit den Fingern geknackt, um die Winde wieder zum Fliessen zu bringen. Weit verbreitet sind auch Schmerzen im Nacken, eine besonders vulnerable Stelle, da die Befürchtung besteht, Blutgefässe des Halses könnten bersten und zu Kopfschmerzen, Übelkeit, Schwindel oder gar zum Tod führen. Die Kambodschaner überprüfen ihr körperliches Befinden ständig auf Blockaden der Winde hin, die in dieserVorstellung so rasch wie möglich behoben werden müssen, um schlimmere Entwicklungen, bis hin zu Ohnmacht oder Tod, verhindern zu können. Abhilfe schaffen zudem sogenanntes Coining (dazu werden Münzen über die Haut gerieben, bis blutige Stellen auf der Haut anzeigen, dass die überschüssigen Winde den Körper verlassen können) sowie Cupping (Schröpfen) (Hinton, Um \& Ba, 2001, S. 403-432). Atemübungen stellen aus dieser von der theravadischbuddhistischen Tradition beeinflussten Sicht ebenso einen Weg dar, die Winde von Blockaden zu befreien.

Die «Person» wird aus vielen «Schichten» bestehend gedacht, die sie beschützen und so für eine ontologische Sicherheit sorgen (Hinton, Hinton et al., 2009). Diese Schichten bestehen aus körperlichen und ausser-körperlichen Zonen. Von innen nach aussen sind dies: eine «stabile Seele», Kühlung des Körpers, normaler Fluss der Winde wie oben beschrieben, ausgeglichene mentale Verfassung, Zahl der erworbenen Karma-Verdienste, Glück, Tattoos, heiliges Wasser, Talismane, Geister des Hauses, Buddha, Ahnen, lokale Gottheiten. Wenn eine dieser Zonen beeinträchtigt ist, besteht die Gefahr, dass die Person von einem Geist oder anderen Kräften angegriffen wird (Hinton, Pich et al., 2005). Körperliche und seelische Krankheiten können durch Eindringen von Geistern in diese erweiterte KörperSphäre verursacht werden, oder dadurch, dass, die oberen Geister‘ den Schutz 
vor Geistern der unteren Sphäre, also vor Dämonen verringern. Dies passiere oft nach einem Fehlverhalten oder moralischem Fehltritt. Die Person werde in der Folge davon in der Gesellschaft geächtet, aber nicht nur sie, sondern auch die ganze Familie sowie die Ahnen der Frau. Deshalb ist es Aufgabe der ganzen Familie, diese Krankheit zu heilen, weil die Krankheit in diesem Verständnis die ganze Familie betrifft. Körperliche Symptome würden als eine von den Ahnen induzierte Krankheit verstanden, die über Generationen weitergegeben werden könne, z. B. weil Ahnen nicht gebührend geehrt wurden (Eisenbruch, 1992, S. 283-316). Oft taucht dieser «Geist» (khmaoch) während des Schlafes auf, was als Albtraum erlebt wird (Hinton, Hinton et al., 2009). Ein Albtraum zeigt an, dass die Sicherheitsschichten einer Person beschädigt sind und «repariert» werden müssen. Dies kann durch Anzünden von Räucherstäbchen vor dem Hausaltar, indem Buddha, die Eltern, die Ahnen, die lokalen Götter und andere Kräfte um Schutz gebeten werden, erreicht werden. Ebenso helfen das Trinken von heiligem Wasser und das Zitieren von Pali-Versen. Der Körper erlangt damit wieder einen gesunden Zustand, der mit einem Gefühl von Frische ("I feel fresh") umschrieben wird (Hinton, Hinton et al., 2009).

Was bedeutet dies nun für die Therapie von M.?

Zunächst einmal scheint es M. gelungen zu sein, sich besser durchzusetzen und sich mehr für sich einzusetzen. Vielleicht fand eine Identifikation mit der Therapeutin statt, die für die Patientin einen «westlichen» Lebensstil verkörperte. Die Patientin befindet sich wie viele junge Kambodschaner und Kambodschanerinnen im Konfliktfeld zwischen traditionellen Lebensweisen, und den Erwartungen, die daran geknüpft sind, sowie den als «westlich» empfundenen Möglichkeiten eigener Interessen und Wünsche wie z. B. eigene Freizeit zu haben, eigene Entscheidungen zu treffen, kurz: sich zu individualisieren. Dies stösst in einer kollektivistisch organisierten Gesellschaft auf Widerstand. Möglicherweise ermutigten die Gespräche M., ihre Wünsche zu verfolgen und nicht aufzugeben, bzw. die Spannungen dieser Konflikte besser auszuhalten. Möglicherweise wurde auch M's Familie angeregt, das Problem gemeinsam zu lösen. Gleichzeitig wurde die Patientin auch darin begleitet, eine ihrer angeschlagenen «Schichten» zu reparieren. Nach Kohte-Meyer (1999) könnten zwei Prozesse angestossen worden sein. Einerseits wird die Therapeutin durch eine Über-Ich-Übertragung eine bedeutsame Person, die die Geschichte der Patientin im Sinne Eriksons «erkennen» kann. Andererseits wird auch ein soziales Über-Ich der Kultur der Therapeutin - und von der Patientin mitangestrebtes - übertragen. Dies ermöglicht der Patientin 
ebenso eine Auseinandersetzung mit unterschiedlichen inneren Repräsentanzen und im besten Fall einerVersöhnung derselben. Für die Therapeutin bedeutet dies, dass sie die oft inkompatiblen Differenzen zweier kultureller Systeme «in einem innerlich aktiven Akt gelten lässt».

Haben die kulturell fremden Patienten auch noch direkt Gewalt erfahren, bekommt im therapeutischen Arbeiten die Bezeugung des Erlebten einen besonderen Stellenwert. Dies wird im Folgenden anhand einer weiteren Fallgeschichte illustriert.

\section{Zeugenschaft als Agens in der psychoanalytischen Arbeit}

Herr S. war knapp drei Jahre bei mir (Sophie Schneider) in Psychotherapie. Er war Mitte Fünfzig, schlank, mit ebenmässigen Gesichtszügen und dunkler Haut. Er sprach gebrochen Schweizerdeutsch mit englischem Akzent und hatte ein stilsicheres Äusseres. Aufgewachsen in Kapstadt, Südafrika, gehörte er der tamilischen Minderheit Sri Lankas an. Der Vater war Alkoholiker und gewalttätig, die Mutter depressiv. In den Townships erlebte er von klein auf rohe Gewalt. Nach dem Tod des Vaters ging er zur Schule und sagte, der Vater sei gestorben - als sei nichts gewesen. Damals war er zwölf Jahre alt. Schneider (2016) nennt dieses Phänomen unter Extremstress treffend «semiotische Dissoziation» (S. 924).

Herr S. kam hernach mit der Mutter und einem Teil der Geschwister in die Schweiz. Noch als Achtzehnjähriger bettnässte er. Dann schlug ihn der Bruder, mit dem er das Bett teilte, zusammen. Nach einer Verkäuferlehre arbeitete er in einem Musikgeschäft und als Gitarrenlehrer, schliesslich kam er als Hotelbesitzer zu Geld. Einer seiner Brüder in Südafrika starb bei einer Messerstecherei. Herr S. wurde kurz darauf von Skin Heads spitalreif geprügelt. Gezeichnet von Gewalt und Verlust, verkaufte er sein Hotel, damals meist unter Alkohol und Cannabis stehend, zu einem Spottpreis und erstattete Anzeige. Die Rechtsstreitigkeiten bestanden über die Therapiezeit hinaus.

Mit Alkohol und Kiffen betäubte Herr S. seine chronischen Schmerzen und Ängste. Zurückgezogen und ohne Arbeit, spielte er die meiste Zeit Gitarre vor dem tonlos laufenden Fernseher. Er erlebte sich als gefühlskalt und fremd. Die Beziehung zu seinen zwei Kindern und deren Müttern war problembehaftet. Seine Freundin lebte in einer anderen Stadt nahe seinem früheren Hotel. Sie war verheiratet und unterstützte ihn finanziell. Ihre «On-Off-Beziehung» lebten sie im Einvernehmen ihres Ehemannes. Besuchte er sie, verliess er kaum je die Wohnung aus Angst, den früheren Betrügern zu begegnen. Meist wohnte er vorübergehend bei anderen, so einmal in einer Heimsauna. Seine Rastlosigkeit erinnert 
an Waldenfels' Worte (2015): «... der Migrant ist Ein- und Auswanderer in einer Person ... dieser doppelte Wandlungsprozess, der bewirkt, dass jemand heimisch wird, wo er fremd war, und fremd wird, wo er heimisch war ... Wir haben es mit einem Prozess zu tun, der sich doppelt beschreiben lässt: als Fremdwerden des Eigenen und Eigenwerden des Fremden» (S. 225 f. [Hervorh. i. O.]).

Seine Zuvorkommenheit mir gegenüber wirkte oft etwas förmlich. Dass starke Anpassungsreaktionen von Migranten in der Gegenübertragung das Gefühl auslösen können, den Patienten nicht zu erreichen, beschreiben auch Kohte-Meyer (1999, S. 79) und Ziegenbein (2008, S. 217). Von einer eigentlichen «emotionalen Sprachlosigkeit» bei Traumatisierten spricht Hirsch (1993). Dabei bezeugte Herr S. wiederholt seine Dankbarkeit darüber, dass ihm hier zugehört und Glauben geschenkt werde. Er fürchtete, den Verstand zu verlieren, oder dass sein «südafrikanisches Temperament» mit seinen Rachephantasien gegenüber den Hotelbetrügern mit ihm durchgehen würde. Es ist für Gewalterfahrene prognostisch wichtig, eine Umwelt vorzufinden, in der das Gewaltsame anerkannt wird (Varvin, 2018, S. 208). Die Analytikerin muss sich emotional davon erfassen lassen, dass das Grauen stattgefunden hat (Kreuzer-Haustein, 2018, S. 227). So übernimmt die Therapie die zentrale Funktion der Zeugenschaft. In diesem Sinne können auch meine an Ämter und Gerichte adressierten Berichte als «Zeugnisse» dessen, was Herrn S. widerfuhr, gedient haben: Sie gaben dem Geschehen über die Therapie hinaus einen «Namen».

Gewisse szenische Episoden mit Herrn S. erfassten mich emotional unmittelbarer und heftiger als verbal Mitgeteiltes: Sein halbvoller Kaffeebecher, der überschwappte; sein überbordender, zerfledderter Aktenberg, den er mitbrachte - Diese Vorgänge hatten die Qualität von enactments und dürften Aspekte seines Innenlebens abgebildet haben, die vielleicht mit Worten nicht mitteilbar waren. Gemäss Freeman ist denn auch eine traumatische Erinnerung «weitgehend sensorisch und bar jeder narrativen Einbettung» (2014, S. 20). So forderten solche embodied memories meine Möglichkeiten zu Holding und Containment immer wieder von neuem heraus. Dass sich Affektintensität und damit potenziell belastende Gegenübertragungsphänomene mit abnehmendem Integrationsgrad traumatischer Erfahrungen verstärken, ist bekannt (z. B. Barwinski (2011, S.137). Insbesondere bei nicht symbolisiert Erinnerbarem beansprucht dies «ein hohes Mass an emotionaler Empfänglichkeit und Bereitschaft des Analytikers, die meist schwer erträglichen Zustände, Affekte und Körperreaktionen überhaupt erst zuzulassen» (Kreuzer-Haustein, 2018, S. 219). Die Begegnung mit Extremtraumatisierten kann das Gegenüber zusätzlich verunsichern, wenn diese darüber hinaus uns 
fremden Kulturen entstammen. Diese doppelte Angst gilt es Ottomeyer und Renner (2008) zufolge besonders zu reflektieren: Denn dabei werden Abwehrleistungen auf den Plan gerufen wie etwa der verleugnende Wunsch, «es möge nicht wahr sein» (S. 165f.).

Herr S. sprach nie konkret über seine Gewalterlebnisse. Dies kann einerseits damit zu tun haben, dass insbesondere schmerzliche Gefühlsqualitäten generell die Grenze sprachlichen Mitteilungsvermögens markieren (Améry et al., 2002, S. 73) und in gewissen Kulturen fast ausschliesslich nonverbal über schmerzhafte Ereignisse kommuniziert wird aufgrund der Befürchtung, durch Aussprechen des Bösen dieses herbeizurufen (Ziegenbein, 2008, S. 221). Herr S. sah als spiritueller Mensch in seinen Albträumen eine Warnung seines gewaltsam verstorbenen Bruders, sich vor den Hotelbetrügern in Acht zu nehmen. Für seine Schwester war es Karma, dass er sein Hotel und Vermögen verloren hatte, da er seinen gewaltsam verstorbenen Bruder auf der Strasse wie einen Hund habe sterben lassen. Auch Herr S. sah seine Krankheit als Strafe für sein menschliches Fehlverhalten; ein Verständniszugang, welcher je nach kulturell-religiösem Hintergrund verbreitet ist (Ziegenbein, 2008, S. 221). Cesko (2018, S. 54) warnt in der interkulturellen Psychotherapie davor, kulturelle Merkmale vorschnell zu pathologisieren oder aber real vorliegende psychische Probleme kulturell zu überfrachten (ÜberKulturalisierung). Was letztlich paranoid und damit pathologisch war und was Ausdruck seiner kulturell geprägten Überzeugung (Magisches Denken, Glaube an schwarze Magie usw.), war mitunter schwer auszumachen.

Herr S. nahm Ämter und Behörden als mehrheitlich negative, ihn schädigende Instanzen wahr. Aufgrund ihrer meist unsicheren psychosozialen Umstände sind Migranten laut Ziegenbein (2008, S. 221) oft misstrauisch und projizieren ihre negativen Erfahrungen auf die Behörden. Waldenfels (2015) meint pointiert, dass fremd jene seien, die im Ernstfall, etwa auf Ämtern oder vor Gericht, «genötigt sind, die Sprache des Anderen zu sprechen, so dass der Andere «das Sagen hat» (S. 225). Reale negative Erfahrungen mit Behörden können Spaltungsprozesse in der Übertragung von Traumatisierten wiederum verstärken und von den begutachtenden Stellen projektiv identifikatorisch ausagiert werden, was so zu teils folgenschweren Fehlurteilen führen kann (Henningsen, 2012, S. 217). Auch in meiner Zusammenarbeit mit dem eingebundenen Helfernetz kam es gehäuft zu Missverständnissen, was vermutlich auf Täter-Opfer-Reinszenierungen mittels gegenseitiger Feindprojektion beruhte (vgl. Pross \& Reddemann, 2009, 164 ff.).

Herr S. erlebte die Untersuchungsmethoden der Invalidenversicherung (IV) als invasiv. Er fühlte sich wieVieh behandelt. Er wurde fotografiert, ihm wurde in den 
Mund geschaut. Er fühlte sich wieVieh behandelt. Am Vortag der Abklärung rauchte er einen Joint zur Beruhigung, worauf ihm die IV eine manifeste Suchterkrankung attestierte, was einen späteren Rentenzuspruch unwahrscheinlich machte. Auf einer Reise nach Südafrika während des IV-Verfahrens wurde er ausgeraubt und um Pass, Geld und Tickets gebracht. Durch die mehrmonatige Verzögerung seiner Ausreise verlor er beinahe seine Schweizer Niederlassungsbewilligung C. Die IV sistierte wegen verletzter Mitwirkungspflicht weitere Abklärungen, und das Sozialamt verlangte eine Neuanmeldung. Ein IV-Rekurs wurde in die Wege geleitet.

Die Psychotherapie bei mir endete, nachdem sich Herr S. auf Druck des Sozialamtes für einen festen Wohnsitz entschied: In der Nähe seiner Freundin fand er durch einen glücklichen Zufall eine günstige Wohnung. Monate später liess er verlauten, dass er einen passenden Psychotherapeuten gefunden hätte. Rückblickend betrachtet war die Arbeit mit Herrn S. ein lohnendes Unterfangen. Sein Befinden und seine Lebensumstände stabilisierten sich merklich. Auch wenn noch viel Chaos in seinem Leben war, so erlebte er sich insgesamt gefestigter in der Beziehung zu seinen Kindern und zu seiner Freundin. Er blieb mehrheitlich suchtmittelfrei und hatte seit Jahren wieder ein eigenes Daheim. Eine definitive Verfügung seitens IV stand bei Therapieabschluss bei mir noch aus.

Es war für mich immer wieder ein zähes Ringen, das ihm Widerfahrene in der Therapie zu bezeugen, ohne selbst detektivisch verfolgend oder ungläubig reserviert zu reagieren - denn die mögliche Begegnung mit dem traumatischen Schrecken macht Angst. Varvin resümiert, «dass der Analytiker sich darauf einlassen muss, mit dem Patienten in psychischen Regionen zu leben, die quälend, bedeutungsleer - und manchmal von Grauen erfüllt sind» (2016, S.840). Wilson und Drožđek (2004, S. 135 ff.) sprechen in diesem Zusammenhang denn auch von einer Abyss-Experience - einer Abgrunderfahrung.

\section{Schlussfolgerung}

Anhand von drei sehr heterogenen Vignetten wurden diverse Aspekte in der Begegnung mit gewalterfahrenen Menschen aus unterschiedlichen Kulturkreisen herausgearbeitet. Dabei beschäftigte uns die Frage, weshalb es auf Seiten der AnalytikerIn zu einer Hemmung, sich darauf einzulassen, kommt. Zusammenfassend spielen dabei drei Gesichtspunkte eine wesentliche Rolle. Der vorgestellte Romanausschnitt fokussierte das Phänomen einer Störung der innerpsychischen Zeitordnung und einer kompensatorischen Überbetonung konkretistischer Details, welche nach unverarbeiteten Gewalterfahrungen auftreten können. Dass subjektiv selbstverständliche Erklärungsmodelle mitunter keine Geltung in 
einem anderen Kulturkreis haben, wurde mit einer Fallstudie aus Kambodscha veranschaulicht. Im Spannungsfeld kultureller Gegensätze wird unsere eigene impliziteWeltauffassung und damit unsere «Vertrautheit mit uns selbst, derWelt und den anderen» bedroht (Hahn, 1997, S. 146). Dabei muss eine innere Spannung, auch das Fremde gelten zu lassen, ausgehalten werden. Denn: «Das Unvertraute bleibt der Stachel im Fleisch der voreiligen Gewissheiten» (Münkler, 1997, S. 26). Anhand einer Psychotherapie mit einem südafrikanischen Mann wurde die analytische Aufgabe erläutert, angesichts des chaotischen Seelenlebens des Patienten immer wieder eine Position der Stabilität zu finden, um so Orientierung zu bieten. In diesem Kräftefeld gilt es, die psychische Realität des Anderen vorbehaltslos zu akzeptieren, damit konsequent Zeugenschaft geleistet werden kann. Mit dem Fremden und dem Risiko des Unsicheren, potenziert in der Begegnung mit Gewalterfahrenen aus einem anderen Kulturraum, geht stets die Angst vor der eigenen Ich-Auflösung einher. Schliesslich tritt man gemäss Bittner (vgl. 1993, S. 208) mit jedem Patienten in eine «gemeinsameWelt» ein. Gadamer spricht von Horizontverschmelzung (2010, S. 311). Und dies bedeutet, dass man in vielen «gemeinsamen Welten» enthalten ist, die nicht nur die Welten der Patienten, sondern auch die eigenen Welten sind. Das heisst: «... das Verstehen des Fremdseelischen verlangt die Aufweichung des allzu kompakten Ich» (Bittner, 1993, S. 209). Diese Verflechtung stellt für die AnalytikerIn eine besondere Herausforderung an deren Leidensfähigkeit und Offenheit dar (vgl. Kreuzer-Haustein, 2018; Varvin, 2018) und birgt zugleich die Möglichkeit, über den eigenen Horizont hinauszuwachsen.

Lassen wir uns auf das Fremde ein, so kommt es zu Grenzverschiebungen, und wir müssen uns ändern. Gehorchen wir der Angst, so werden wir unsere Grenzen verstärken und befestigen. Dass Fremde wird dann zum Feind, der mit Gewalt abgewehrt werden muss und dessen Gegenwart uns ängstlich und starr macht. (Erdheim, 1993, S. 180)

\section{Anmerkungen}

1 Besonderer Dank gilt Daniel Strassberg, der das dem Artikel zugrunde liegende Seminar mitgestaltete und während des Schreibprozesses immer wieder wertvolle Hinweise gab.

\section{Literatur}

Améry, J., Scheit, G., \& Heidelberger-Leonard, I. (Hrsg.) (2002). Werke:/ Jean Améry. Hrsg. von Irene Heidelberger-Leonard; Bd. 2. Jenseits von Schuld und Sühne. Stuttgart: Klett-Cotta. 
Barwinski, R. (2011). Die erinnerte Wirklichkeit: Zur Bedeutung von Erinnerungen im Prozess der Traumaverarbeitung (2. Aufl.). Psychotraumatologie, Psychotherapie, Psychoanalyse: Vol. 24. Kröning: Asanger.

Beil, C. (2013). Israel: Die Geschichte des jüdischen Staates. Geo Epoche: Vol. 61. Hamburg: Gruner + Jahr.

Bittner, G. (1993). Psychoanalytisches Verstehen als Scheitern des Eigenen am Fremden. In U. Streeck (Hrsg.), Leben lernen: Vol. 88. Das Fremde in der Psychoanalyse. Erkundungen über das «Andere» in Seele, Körper und Kultur. München: Verlag J. Pfeiffer.

Cesko, E. (2018). Transkulturelle Psychotherapie. Neue Perspektiven in der klinischen Anwendung. Psychotherapie-Wissenschaft, 8(2), 51-56.

Chandler, D. P. (1991). The tragedy of Cambodian history: Politics, war, and revolution since 1945. New Haven: Yale University Press.

Eisenbruch, M. (1992). The Ritual Space of Patients and Traditional Healers in Cambodia. Bulletin de l'École française d'Extrême-Orient, 79(2), 283-316, from https://www.persee.fr/docAsPDF/befeo_0336-1519_1992_num_79_2_1882. pdf.

Erdheim, M. (1993). Das Fremde - Totem und Tabu in der Psychoanalyse. In U. Streeck (Ed.), Leben lernen: Vol. 88. Das Fremde in der Psychoanalyse. Erkundungen über das «Andere» in Seele, Körper und Kultur (167-183). München: Verlag J. Pfeiffer.

Freeman, M. (2014). Nachträglichkeit, traumatisch und nicht-traumatisch: Erinnerung, Erzählung und das Mysterium der Ursprünge. In C. E. Scheidt (Hrsg.), Narrative Bewältigung von Trauma und Verlust ( $1^{\text {st }}$ ed.). s.l.: : :

Freud, S. (2000). Jenseits des Lustprinzips. $n$ Studienausgabe Band III Psychologie des Unbewussten. Frankfurt am Main: Fischer.

Gadamer, H. G. (2010). Gesammelte Werke: Band 1: Hermeneutik I: Wahrheit und Methode: Grundzüge einer philosophischen Hermeneutik (7., überarb. Aufl.). Gesammelte Werke:/Hans-Georg Gadamer ; Bd. 1. Tübingen: Mohr Siebeck. I ?

Gutwinski-Jeggle, J. (2017). Unsichtbares sehen - Unsagbares sagen: Unbewusste Prozesse in der psychoanalytischen Begegnung (Originalausgabe). Bibliothek der Psychoanalyse. Gießen: Psychosozial-Verlag.

Hahn, A. (1997). Partizipative Identitäten. In H. Münkler \& B. Ladwig (Eds.), Studien und Materialien der Interdisziplinären Arbeitsgruppe. Die Herausforderung durch das Fremde der Berlin-Brandenburgischen Akademie der Wissenschaften. Furcht und Faszination. Facetten der Fremdheit. Berlin: Akad.-Verl. 
Henningsen, F. (2012). Psychoanalysen mit traumatisierten Patienten: TrennungKrankheit-Gewalt. Fachbuch Klett-Cotta. Stuttgart: Klett-Cotta.

Heuveline, P. (2017). Households and family processes. In K. Brickell \& S. Springer (Eds.), The handbook of contemporary Cambodia. London, New York: Routledge.

Hinton, D. E., Hinton, A. L., Pich, V., Loeum, J. R., \& Pollack, M. H. (2009). Nightmares among Cambodian refugees: the breaching of concentric ontological security. Culture, medicine and psychiatry, 33(2), 219-265.

Hinton, D., Um, K., \& Ba, P. (2001). Kyol Goeu (“Wind Overload”) Part I: A Cultural Syndrome of Orthostatic Panic among Khmer Refugees. Transcultural psychiatry, 38(4), 403-432.

Hinton, D. E., Pich, V., Chhean, D., \& Pollack, M. H. (2005). “The ghost pushes you down": sleep paralysis-type panic attacks in a Khmer refugee population. Transcultural psychiatry, 42(1), 46-77.

Hirsch, M. (1993). Das Fremde als unassimiliertes Objekt. In U. Streeck (Ed.), Leben lernen: Vol. 88. Das Fremde in der Psychoanalyse. Erkundungen über das «Andere» in Seele, Körper und Kultur. München: Verlag J. Pfeiffer.

Kanafāni, Ġ. (1992). Rückkehr nach Haifa: Roman aus Palästina (1. Aufl., für die Taschenbuchausg. überarb). Arabische Literatur im Lenos-Verlag: Vol. 11. Basel: Lenos-Verl.

Kimura, B. (Ed.) (1995). Zwischen Mensch und Mensch: Strukturen japanischer Subjektivität. Darmstadt: Wiss. Buchges.

Kohte-Meyer, I. (1999). Spannungsfeld Migration: Ich-Funktionen und Ich-Identität im Wechsel von Sprache und kulturellem Raum. In F. Pedrina (Ed.), Kultur, Migration, Psychoanalyse. Therapeutische Konsequenzen theoretischer Konzepte ; eine Vortragsreihe des Psychoanalytischen Seminars Zürich. Tübingen: Ed. diskord.

Kraus, W. (2014). Arbeit am Unerzählbaren. Narrative Identität und die Nachtseite der Erzählbarkeit. In C. E. Scheidt (Ed.), Narrative Bewältigung von Trauma und Verlust ( $1^{\text {st }}$ ed.). s. l.: Schattauer GmbH Verlag für Medizin und Naturwissenschaften.

Kreuzer-Haustein, U. (2018). Geflüchtete und Traumata. Psyche, 72(3), 216-230, from https://www.psyche.de/article/ps_2018_03_0216-0230_0216_01.pdf.

Kristeva, J. (1990). Fremde sind wir uns selbst (Dt. Erstausg., 1. Aufl.). Edition Suhrkamp: $1604=$ n.F., 604. Frankfurt am Main: Suhrkamp.

Küchenhoff, J. (2015). Interkulturelle Gewalt und interkulturelle Übergangsräume. Konstruktion und Dekonstruktion des Fremden. In O. Gutjahr (Ed.), Frei- 
burger literaturpsychologische Gespräche: Vol. 34. Interkulturalität. Konstruktionen des Anderen ; [enthält ... Beiträge, die auf der psychoanalytischliteraturwissenschaftlichen Arbeitstagung zum Thema Interkulturalität am 24.-25. Januar 2015 in Freiburg vorgestellt und diskutiert wurden. Würzburg: Königshausen \& Neumann.

Lorenzer, A. (1983). Sprache, Lebenspraxis und szenisches Verstehen in der psychoanalytischen Therapie. Psyche, 37(2), 97-115, from https://www.psyche. de/article/ps_1983_02_0097-0115_0097_01.pdf.

Münkler, H. (1997). Dimensionen der Fremdheit. In H. Münkler \& B. Ladwig (Eds.), Studien und Materialien der Interdisziplinären Arbeitsgruppe. Die Herausforderung durch das Fremde der Berlin-Brandenburgischen Akademie der Wissenschaften. Furcht und Faszination. Facetten der Fremdheit. Berlin: Akad.-Verl.

Nakagawa, K. (2006). More Than White Cloth?: Women's Rights in Cambodia:Cambodian Defenders Project.

Ottomeyer, K. \& Renner, W. (2008). Die doppelte Befremdung: Diagnostik und wirksame Psychotherapie bei traumatisierten Flüchtlingen. In S. GolsabahiBroclawski \& T. Heise (Eds.), Das transkulturelle Psychoforum: Vol. 15. Von Gemeinsamkeiten und Unterschieden. 1. Kongress der Transkulturellen Psychiatrie im Deutschsprachigen Raum, 6.-9. September 2007, Universität Witten/Herdecke (pp. 165-175). Berlin: VWB Verl. für Wiss. und Bildung.

Ovesen, J., \& Trankell, I.-B. (2010). Cambodians and their doctors: A medical anthropology of colonial and post-colonial Cambodia. NIAS monographs:Vol. 117. Copenhagen: NIAS Press.

Pross, C., \& Reddemann, L. (2009). Verletzte Helfer: Umgang mit dem Trauma: Risiken und Möglichkeiten, sich zu schützen. Leben lernen:Vol.222. Stuttgart: Klett-Cotta.

Schneider, C. (2016). Das Ferne, das uns nahe ist. Psyche, 70(9), 923-948, from https://www.psyche.de/article/ps_2016_09_0923-0948_0923_01.pdf.

Varvin, S. (2016). Asylsuchende und Geflüchtete: ihre Situation und ihre Behandlungsbedürfnisse. Psyche, 70(9), 825-855, from https://www.psyche.de/ article/ps_2016_09_0825-0855_0825_01.pdf.

Varvin, S. (2018). Unser Verhältnis zu Flüchtlingen: Zwischen Mitleid und Entmenschlichung. Psyche, 72(3), 194-215, from https://www.psyche.de/ article/ps_2018_03_0194-0215_0194_01.pdf.

Waldenfels, B. (1999). Topographie des Fremden (2.Aufl.). Studien zur Phänomenologie des Fremden: / Bernhard Waldenfels ; 1. Frankfurt am Main: Suhrkamp. / ? 
Waldenfels, B. (2015). Fremdheit innerhalb und ausserhalb unserer eigenen Kultur. In O. Gutjahr (Ed.), Freiburger literaturpsychologische Gespräche: Vol. 34. Interkulturalität. Konstruktionen des Anderen; [enthält ... Beiträge, die auf der psychoanalytisch-literaturwissenschaftlichen Arbeitstagung zum Thema Interkulturalität am 24.-25. Januar 2015 in Freiburg vorgestellt und diskutiert wurden (pp. 213-231). Würzburg: Königshausen \& Neumann.

Wilson, J.P. \& Drožđek, B. (2004). Broken spirits. The treatment of traumatized asylum seekers, refugees, war and torture victims (pp. 135-138). New York: Brunner-Routledge.

Winnicott, D. W. (1991). Die Angst vor dem Zusammenbruch. Psyche, 45(12), 11161126, from https://www.psyche.de/article/ps_1991_12_1116-1126_1116_01. pdf.

Ziegenbein, M. (2008). Psychiatrische Begutachtung im interkulturellen Feld. In S. Golsabahi-Broclawski \& T. Heise (Eds.), Das transkulturelle Psychoforum: Vol. 15. Von Gemeinsamkeiten und Unterschieden. 1.Kongress der Transkulturellen Psychiatrie im Deutschsprachigen Raum, 6.-9. September 2007, Universität Witten/Herdecke (pp. 213-224). Berlin: VWB Verl. für Wiss. und Bildung.

\section{Angaben zu den Autoren und Autorinnen}

Elizabeth Högger Klaus, lic. phil., 1977. Psychoanalytische Psychotherapeutin in eigener Praxis und Co-Präsidentin Entresol.

Sophie Schneider, lic. phil., 1968. Psychoanalytische Psychotherapeutin PSZ, PSB und EFPP, Oberpsychologin bei Clienia Gruppenpraxen AG Männedorf, Vorstandsmitglied Entresol.

Michael Niebler, Dr. med., 1983. Psychiater in freier Praxis und Vorstandsmitglied Entresol. 